\title{
Reversing tumor stemness via orally targeted nanoparticles achieves efficient colon cancer treatment
}

\author{
Jiaqi Xu ${ }^{1,2}$, Yinlong Zhang ${ }^{1 *}$, Junchao $\mathrm{Xu}^{1,2}$, Meifang Wang ${ }^{1,3}$, Guangna Liu ${ }^{1,3}$, Jing Wang ${ }^{1,2}$, Xiao \\ Zhao ${ }^{1}$, Yingqiu Qi ${ }^{4}$, Jian Shi ${ }^{1}$, Keman Cheng ${ }^{1}$, Yao $\mathrm{Li}^{1}$, Sheng $\mathrm{Qi}^{5^{*}}$, Guangjun Nie ${ }^{1,2^{*}}$
}

${ }^{1}$ CAS Key Laboratory for Biomedical Effects of Nanomaterials \& Nanosafety, CAS Center for Excellence in Nanoscience, National Center for Nanoscience and Technology, Beijing 100190, China ${ }^{2}$ Center of Materials Science and Optoelectronics Engineering, University of Chinese Academy of Sciences, Beijing 100049, China

${ }^{3}$ College of Pharmaceutical Science, Jilin University, Changchun 130021, China ${ }^{4}$ School of Basic Medical Sciences, Zhengzhou University, Henan 450001, China ${ }^{5}$ School of Pharmacy, University of East Anglia, Norwich, Norfolk, NR4 7TJ, UK

*Corresponding authors:

Guangjun Nie, email: niegj@nanoctr.cn; Sheng Qi, email: Sheng.Qi@uea.ac.uk Yinlong Zhang, email: zhangyl@,nanoctr.cn 


\section{Abstract}

The acquisition of stemness in colorectal cancer (CRC) attributed to the recurrence and metastasis in CRC treatment. Therefore, targeting the stemness of CRC forms a basis for the development of novel therapeutic approaches. However, the pain and systemic side effect from long-term of venipuncture injection remain great challenges to neoplastic treatment. Here, we introduce an oral drug delivery system for sustained release of BMI-1 inhibitor (PTC209) that reverse the stemness of CRC to overcome these obstacles. In this system, nanoparticles modified with hyaluronic acid (HA) showed high-affinity to CD44 / CD168 overexpressed-CRC cells, and efficiently targeted to tumor site in a metastatic orthotropic colon cancer mouse model by oral administration. Significantly, the observed tumor growth inhibition is accompanied by decreased expression of stemness markers in the tumor tissues. Furthermore, HA-NPsPTC209 also significantly prevented metastasis to the gastrointestinal system, while failing to exhibit acute side effects. In summary, we have developed an orally active, easily synthesized nanomedicine that shows promise for the treatment of colon cancer.

Keywords: Colorectal cancer; Oral drug delivery; Stemness; PTC209 


\section{Introduction}

Colorectal cancer (CRC), the fourth leading cause of cancer-related death, is one of the most common malignancies worldwide ${ }^{1,2}$. Unfortunately for CRC victims, conventional chemotherapy and radiation provide only limited treatment efficacy in the clinic $^{3}$. Therefore, there is an urgent need for new therapeutic strategies to treat CRC. Increasing evidence indicates that cancer stem cells (CSCs), a subtype of cells within the tumor that exhibit stem-like behavior, including tumor-initiation, invasion, metastasis, and chemotherapy resistance, are responsible for the failure of current CRC treatments ${ }^{4-6}$. Thus, selectively reversing the stemness of CSCs is likely to provide a promising strategy for CRC treatment. Indeed, several drugs targeting CSCs have been investigated in clinical trials. These drugs include BBI608 (Phase 3), PTC-596 (Phase 1), and Salinomycin (Phase 1/2). Several preclinical studies have suggested that these agents greatly decline tumor progression alone or in combination with existing treatment methods ${ }^{7-}$ ${ }^{9}$. Among these drugs, PTC209, a specific inhibitor of BMI-1 (B cell-specific Moloney murine leukemia virus integration site 1), a major regulator of self-renewal capacity in several tissues, can reverse the stemness of CSCs and has been applied to the treatment of colon cancer, myeloma and acute myeloid leukemia ${ }^{10-12}$. Importantly, BMI-1 is overexpressed in CRC and is considered an indicator of malignancy, correlating with tumors metastasis, epithelial mesenchymal transition (EMT), and chemotherapy resistance $^{13-15}$. The presence of a biological function of BMI-1 in tumors implies that patients may benefit from BMI-1-targeting agents. Therefore, reducing the stemness of CSCs by efficient delivery of PTC209 presents an attractive strategy for CRC treatment.

With the advantages of high patient compliance, convenience, and cost-effectiveness, the oral route is widely considered the most popular drug administration method ${ }^{16}$. However, oral drug delivery faces several challenges, such as the poor solubility of drugs, the complex gastrointestinal environment, and nonspecific drug distribution in the gastrointestinal tract ${ }^{17}$. With the recent developments in nanomedicine, nanoparticle-based oral drug delivery systems are now possible, providing the potential to enhance permeation through intestinal barrier, alleviate adverse systemic effects, and improve treatment efficiency $^{18-20}$. In addition, targeting ligand-modified drug delivery systems aimed at improving therapeutic outcomes of gastrointestinal tract diseases have been developed ${ }^{21-24}$. Extensive clinical evidence has demonstrated that CD44 and CD168 are overexpressed in several malignant tumors and can be utilized for targeted drug design ${ }^{25,26}$. Hyaluronic acid (HA), a natural polysaccharide composed of N-acetyl- 
D-glucosamine and D-glucuronic acid, has been rigorously validated as a target of CD44 and CD168 27 . Meanwhile, HA possesses unique advantages such as low toxicity, good biocompatibility, biodegrade ability, and low cost, all of which benefit an oral drug delivery system ${ }^{28}$.

In the present study, we synthesized a poly (ethylene glycol)-poly (lactide-coglycolide) (PEG-PLGA) nanoparticle by a double emulsion method to generate an oral delivery vehicle for the BMI-1 inhibitor, PTC209. To equip the particle with targeting ability, we functionalized its surface with the CD44 / CD168 targeting ligand HA (HA-NPs-PTC209; Figure 1A). We assessed the formulation's anti-tumor activity in a CT26 orthotropic colon tumor model. Ex vivo pharmacokinetic and distribution experiments showed that HA-NPs-PTC209 was able to efficiently accumulate in tumor tissue. The results of in vivo antitumor experiments demonstrated that HA-NPs-PTC209 can significantly suppress CT26 orthotropic xenograft growth and metastasis. Meanwhile, investigation of the mechanism of the nanoparticle's action in vitro and in vivo indicated that HA-NPs-PTC209 down-regulates the expression of the stemness markers Sox-2 and Oct-4. This simple, orally active nanoparticle vehicle for targeted delivery of BMI-1 inhibitor is a promising strategy to reverse the stemness of CSCs and achieve long term efficacy against colon cancer (Figure 1B). Moreover, this approach may also be applied to improve the outcomes of other therapeutics.

\section{Methods}

\section{Preparation of HA-NPs-PTC209}

Empty PEG-PLGA nanoparticles (NPs), HA-NPs and HA-NPs-PTC209 were synthesized by the improved double emulsion (w/o/w) method based on our previous work ${ }^{29}$. Briefly, $10 \mathrm{mg} \mathrm{NH}_{2}$-PEG-PLGA and mPEG-PLGA (1:1) (75:25, MW-5000:15000, Xi'an Ruixi Biological Technology Co., Ltd), dissolved in $900 \mu \mathrm{L}$ methylene chloride, and PTC209 (T2345-50 mg, TargetMol) dissolved in $100 \mu \mathrm{L}$ DMSO/methanol (1:9) were transferred to a $10 \mathrm{~mL}$ centrifuge tube and then $200 \mu \mathrm{L}$ water was also added. Next, the mixture was emulsified by ultrasonic generator (Scientz-IID, Ningbo Scientz Biotechnology Co., Ltd., China) for 3 mins at a power of $25 \%$ to obtain the primary emulsion. Then $2 \mathrm{~mL}$ of $2 \%$ polyvinyl alcohol (PVA) was added and emulsified by ultrasonic generator at a power of $30 \%$ for another 5 mins. The emulsion was then slowly added dropwise into $10 \mathrm{~mL} 0.6 \%$ PVA and stirred for 10 mins at 
room temperature. After vacuum evaporation to eliminate the organic solvent, the nanoparticles were collected by centrifugation at $18,516 \mathrm{~g}$ for $20 \mathrm{~min}$ and washed twice with distilled water. To endow the nanoparticles with a targeting capacity, the carboxyl group of HA(MW:6000, Bloomage Freda Biopharm Co.,Ltd) was first activated with N-hydroxysuccinimide (H6231-100g, Macklin) and ethyl-3-(3-dimethylaminopropyl) carbodiimide hydrochloride (N808856-100g, Macklin) at a molar ratio of 1:10:10 in water at room temperature for $24 \mathrm{~h}$. The amino-modified NPs-PTC209 were then conjugated with activated HA at a molar ratio of 1:10 at room temperature for $12 \mathrm{~h}$. The targeted nanoparticles were collected by centrifugation at 18,516g for 20 mins and washed twice with distilled water. For in vitro and in vivo fluorescence imaging, Cy5.5 (A8103-5 MG, ApexBio) instead of PTC209 was added during the preparation process.

\section{Characterization of nanoparticles}

To determine the successful conjugation of HA onto the nanoparticles, samples of HA, NPs and HA-NPs were mixed with potassium bromide and compressed into transparent pellets for FT-IR spectrometry (Spectrum One, Perkin Elmer, USA) detection. The scanning range was between 4000 and $400 \mathrm{~cm}^{-1}$.

The size and surface charge of the NPs, HA-NPs and HA-NPs-PTC209 were evaluated using ZetaSizer (Zetasizer Nano ZS, Malvern Instruments, UK). Each sample was measured three times. The surface morphology was determined by TEM (HT-7700, JEOL, Japan). In brief, $10 \mu \mathrm{L}$ samples of appropriate concentrations were dropped onto 200-mesh copper grids that had been pre-treated with ultraviolet (UV) light to reduce static electricity. After a 5 min incubation, the solution was removed and the grid was negatively stained with a $1 \%$ sodium phosphotungstate solution.

HA-NPs-PTC209 was suspended in distilled water at $37^{\circ} \mathrm{C}$ and the size and zeta potential were determined at 0, 1 and 2 days by dynamic light scattering (DLS). To measure the content of PTC209 in the prepared nanoparticles, the nanoparticles were lyophilized. Acetonitrile (A998-4, Fisher Chemical) was added into the dry nanoparticles under vigorous stirring. The mixture was centrifuged at 10,000 $g$ for 5 min. The amount of PTC209 was determined by measuring the absorbance at $235 \mathrm{~nm}$ using an HPLC system (SPD-20A, SHIMADZU, Japan). The mobile phase (acetonitrile/water: 70 / 30 containing $0.1 \%$ trifluoroacetic acid) was delivered at rate of $1.0 \mathrm{~mL} / \mathrm{min}$ for $10 \mathrm{mins}$. The concentration of PTC209 was 
determined by the peak area at the retention time of 2.7 mins by C18 column (15749, Thermo Fisher Scientific). Encapsulation efficiency and loading efficiency of PTC209 was calculated by the following formula:

Encapsulation Efficiency $=($ weight of loaded drug $) /($ weight of initially added drug $) \times 100 \%$

Loading Efficiency $=($ weight of loaded drug in the nanoparticle $) /($ weight of the nanoparticle $) \times 100 \%$

For in vitro drug release, HA-NPs-PTC209 (20 mg) was suspended in PBS (1 mL, pH 1.2 and pH 6.8) and transferred to dialysis bags (MWCO: $3000 \mathrm{Da}$, Spectrum) placed in $30 \mathrm{~mL}$ corresponding $\mathrm{pH}$ PBS containing $1 \%$ Tween 80 with stirring at $200 \mathrm{rpm} / 37^{\circ} \mathrm{C} .1 \mathrm{~mL}$ of the buffer solution was replaced with $1 \mathrm{~mL}$ fresh PBS at regular intervals. The concentration of PTC209 was determined using HPLC.

\section{Cytotoxicity assay}

CT26 cells (Donated by Prof. Qin Zhihai's Group, Institute of Biophysics, CAS) and CT26-luc cells were grown in RPMI Medium 1640 (350-000-CL, WISENT), supplemented with 10\% fetal bovine serum $(04-010-1 \mathrm{~A}, \mathrm{BI})$, at $37^{\circ} \mathrm{C}$ in a humidified atmosphere of $95 \%$ air and $5 \% \mathrm{CO}_{2}$. For cytotoxicity assays, CT26 cells were seeded on 96-well plates at a density of 6,000 cells per well. After $12 \mathrm{~h}$, the cells were treated with medium containing 1\% FBS and the various drugs or drug-loaded NPs for $24 \mathrm{~h}$ and 48 h. The proportion of viable cells was evaluated using the CCK-8 assay (CK04-500, Dojindo).

\section{Confocal microscopy and flow cytometry analysis}

CT26 cells were seeded on a $35 \mathrm{~mm}$ borosilicate chambered cover glasses (801002, Nest) at a density of $1 \times 10^{4}$ cells $/ \mathrm{mL}$ and incubated with HA-NPs-Cy5.5 or NPs-Cy5.5 for $4 \mathrm{~h}$. The cells were then gently washed 3 times with PBS and fixed in 4\% paraformaldehyde for 20 mins. Two-photon excitation confocal microscopy images were acquired with an Olympus FV 1000 equipped with a femtosecond Ti:sapphire laser. Excitation / emission wavelengths of 675 / $705 \mathrm{~nm}$ were used to detect the Cy5.5 signal of nanoparticles.

To evaluate the targeting ability of HA-NPs-PTC209, CT26 cells were seeded on 6-well plates at a density of $1 \times 10^{5}$ cells per well and incubated with HA-NPs-Cy5.5 or NPs-Cy5.5 for $2 \mathrm{~h}$. Subsequently, the cells were washed with PBS and harvested for flow cytometry analysis (Accuri C6, BD, USA). 


\section{Cancer stem-like spheroid formation assay}

We used a spheroid formation assay to evaluate the stemness of CT26 tumor cells ${ }^{30}$. The cells were plated on Ultra-Low Attachment Surface 24 well-plates (3473, Corning) in serum-free 1640 medium supplemented with B27 (1:50, 17504044, Invitrogen), EGF (20 ng / mL, AF-100-15, Peprotech) and $\beta$-FGF (10 ng / mL, 100-18B, Peprotech) at a density of 800 cells per well. When the number of cells in a spheroid were more than 10, it was considered to have formed a clone sphere. After treatment with different drug formulations for 7 days, the diameter of the sphere was recorded in the microscopy images.

\section{Wound scratch assay}

CT26 tumor cells were plated on 6 -well plates at a density of $2 \times 10^{5}$ cells per well. When the tumor cells grew to approximately $80 \%$, a straight line was drawn in the middle of the plate and the plate was washed 3 times with PBS to remove the dislodged cells. After incubation with different drug formulations in serum-free medium, the numbers of cells that migrated into the scratched region were determined by microscopy.

\section{CD44 and CD133 expression}

CT26 tumor cells were seeded on 6 -well plates at the density of $2 \times 10^{4}$ cells per well. After incubating the samples for $24 \mathrm{~h}$, the cells were treated with PTC209, NPs-PTC209 or HA-NPs-PTC209 at a concentration of $250 \mathrm{nM}$ PTC209 for $48 \mathrm{~h}$. We then stained the cells with PE-conjugated anti-CD44 (orb198169, Biorbyt) and PE-conjugated anti-CD133 (372804, BioLegend) according to the manufacturer's respective instructions. The CD44 and CD133 expression were assessed by flow cytometry.

\section{Establishment of orthotopic murine colon cancer model}

Female BALB/c mice (18-20 g) were obtained from Vital River Laboratory Animal Technology Co. China and maintained in a $12 \mathrm{~h}$ light-dark cycle at $22^{\circ} \mathrm{C}$ and food and water ad libitum. All animal protocols were approved by the Institutional Animal Care and Use Committee of National Center for Nanoscience and Technology. The stably expressing luciferase CT26 cells were established through a lentivirus transduction system and screened using puromycin (J593-25MG, Amresco), as described previously $^{31}$. We then established an orthotopic colon cancer model according to published work ${ }^{32}$. Briefly, 5 
$\times 10^{4}$ of CT26-luc tumor cells dispersed in a $10 \mu \mathrm{L}$ mixture of PBS, Matrigel matrix and India ink, at a ratio of 1:2:1, was surgically injected into the submucosal layer of the cecum wall using a $1 \mathrm{~mL}$ syringe with a 29 G BD Insulin Syringe. The cecum was gently returned to the original location and the cecotomy was closed with 7-0 silk sutures. During this process, the India ink was used to confirm the mixture was successfully injected into the submucosal layer.

\section{Ex vivo fluorescence imaging}

To evaluate the tumor targeting ability of HA-NPs-PTC209, an orthotopic colon tumor model was established using 6-8-week-old BALB/c mice. The mice were fasted but allowed free water for $24 \mathrm{~h}$ prior to the experiment to avoid food spontaneous fluorescence. After administration of Cy5.5-labled HA-NPsPTC209 or NPs-PTC209 for $8 \mathrm{~h}$ and $24 \mathrm{~h}$, the mice were sacrificed and the cecum-colon organs were dissected and washed three times for fluorescence signal detection using an IVIS system and Living Image Software (Spectrum CT, PerkinElmer, USA).

\section{Bio-distribution of PTC209 in tumor and gut tissue}

The established orthotopic colon tumor model described above was used to investigate the bio-distribution of different drug formulations of PTC209. A PTC209 Tween 80 solution, NPs-PTC209 or HA-NPsPTC209 were orally administrated to the fasting CT26 tumor-bearing mice (18-20 g). The dose of PTC209 was equivalent to $30 \mathrm{mg} / \mathrm{kg}$ body weight. $8 \mathrm{~h}$ or $24 \mathrm{~h}$ after drug administration, three mice in each group were lethally anesthetized and gastrointestinal samples were dissected and weighted. The obtained organs were homogenized in $2 \mathrm{~mL}$ PBS (pH 7.4) using a tissue homogenizer at $4{ }^{\circ} \mathrm{C}$ for $1 \mathrm{~min}$. 3 volumes of acetonitrile were added to the homogenate to precipitate the protein and extract PTC209. After centrifugation of the organic-water phases of the tissue homogenates $(10,000 \mathrm{~g}$ for $10 \mathrm{~min})$, the solution was dried and then dissolved in $500 \mu \mathrm{L}$ acetonitrile for HPLC detection.

\section{In vivo antitumor efficacy}

Orthotopic CT26-luc tumor-bearing mice were randomly divided into 5 different experimental groups. The mice were treated orally with saline, empty NPs, PTC209 solution in 1\% Tween 80, NPs-PTC209 or HA-NPs-PTC209 (equivalent to $30 \mathrm{mg}$ / $\mathrm{kg}$ bodyweight) every two days for a total of 14 days. Tumor size was detected by measuring the bioluminescence using an IVIS system and Living Image Software 
every 7 days for three times. After treatment for 14 days, the mice were sacrificed and the intestinal system was excised to observe the metastasis conditions. The tumor tissues were then fixed in formalin and used for immunohistochemical analysis.

\section{Western blot analysis and immunohistochemistry}

Western blot and immunohistochemical (IHC) staining of tumor tissues were performed using antiSox2 antibody (1:1000, ab97959, Abcam), anti-Oct-4 antibody (1:500, ab18976, Abcam) and anti- $\beta$-actin antibody (1:5000, ab8226, Abcam) to estimate the relative protein expression levels in the different treatment groups.

\section{Statistical analysis}

Statistical analysis was conducted using SPSS 17.0 statistical analysis software by the independent Student's T test for comparison of two groups, and one-way ANOVA for multiple groups. All the data are presented as the mean $\pm \mathrm{SD} . P$ values of $<0.05$ were considered statistically significant.

\section{Results and discussion}

\section{Characterization of HA-NPs-PTC209}

To fabricate HA-NPs-PTC209, we chose the amphiphilic copolymer PEG-PLGA, a conjugation of two biodegradable monomers (PEG and PLGA) that have been approved for clinical use by the US Food and Drug Administration (FDA), to encapsulate the BMI-1 inhibitor, PTC209, by the improved double emulsion method (NPs-PTC209) ${ }^{29}$. Since HA can target CD44 / CD168 which are overexpressed on the surface of colon tumor cells, we coated amino-modified NPs-PTC209 with HA by covalent conjugation to form HA-NPs-PTC209. To verify the successful conjugation of HA, we performed FT-IR spectrum analysis. The stretching vibration $\left(3522 \mathrm{~cm}^{-1}\right)$ of the hydroxyl groups in HA-NPs was strengthened compared with NPs $\left(3441 \mathrm{~cm}^{-1}\right)$. Meanwhile, there is a stretching vibration $\left(1648 \mathrm{~cm}^{-1}\right)$ from $-\mathrm{COOH}$ in both HA and HA-NPs (Figure 2A). We next characterized the morphology, hydrodynamic diameter and surface charge of the nanoparticles by transmission electron microscopy (TEM) and dynamic light scattering (DLS). The average hydrodynamic diameter of HA-NPs-PTC209 was $232 \mathrm{~nm}$ which was larger than empty NPs with a diameter of about $200 \mathrm{~nm}$ (Figure 2B-D). The zeta potential of NPs, HA-NPs and HA-NPs-PTC209 were respectively $-2.3 \mathrm{mV},-20 \mathrm{mV}$ and $-19 \mathrm{mV}$ (Figure 2B). TEM images of NPs and 
HA-NPs-PTC209 revealed that both possess typical spherical morphology. The HA-NPs-PTC209 exhibited a 5 nm layer of HA (Figure 2C\&D).

To explore the drug-loading capacity, different ratios of copolymer to drug were selected. With increasing amounts of PTC209, the drug loading content increased while the drug encapsulation efficiency first increased and then decreased. When the ratio was 10:0.25, the drug encapsulation efficiency was highest (62.8\%), which was used for subsequent experiments (Table S1). We next evaluated the stability of HANPs-PTC209 by incubating the particles in PBS for three days at $37^{\circ} \mathrm{C}$. The particle size and zeta potential changed only slightly over the course of three days (Table S2). According to the literature, the retention times of oral drugs are about half an hour in the stomach, 6-10 hours in the small intestine, and in the colon until defecation ${ }^{33}$. To evaluate the drug release profiles of HA-NPs-PTC209 in the gastrointestinal system, we measured the accumulative PTC209 release at $\mathrm{pH} 1.2$ for $3 \mathrm{~h}$, mimicking gastric environment and at $\mathrm{pH} 6.8$ for $72 \mathrm{~h}$, mimicking intestinal environment. As the results in Figure S1 show, HA-NPs-PTC209 released about $26 \%$ of the drug within the first $1 \mathrm{~h}$ in $\mathrm{pH} 1.2$ and about $66 \%$ and $79 \%$ of the drug in $\mathrm{pH} 6.8$ in $24 \mathrm{~h}$ and $48 \mathrm{~h}$, respectively.

\section{In vitro tumor targeting and antitumor activity}

To evaluate the targeting specificity of HA-NPs-PTC209, we observed CT26 tumor cells by confocal laser scanning microscopy (CLSM) after incubating them for $4 \mathrm{~h}$ with Cy5.5-labled NPs-PTC209 or Cy5.5-labeled HA-NPs-PTC209. As expected, the HA decorated nanoparticles showed a greater tumor targeting ability than the non-targeting NPs-PTC209. When we added $1 \mathrm{mM}$ free HA to the HA-NPsPTC209-treated groups, the fluorescence intensity significantly decreased, indicating the targeting capability of HA-NPs-PTC209 was mediated by HA, which competitively inhibited the particle binding (Figure 3A). We also use flow cytometry analysis (FCM) to investigate cellular uptake or targeting after incubation for $2 \mathrm{~h}$ with different drug formulations. The results were consistent with those of the CLSM experiments in that HA-NPs-PTC209 showed more extensive internalization or targeting than the other groups (Figure 3B). As in the CLSM experiments, we added free HA to HA-NPs-PTC209 treated samples and found a significant decline in the fluorescence intensity. Together, the results indicate that the HA-coating of nanoparticles can efficiently enhance cellular uptake or targeting of CT26 tumor cells in vitro. 
To investigate whether the encapsulated BMI-1 inhibitor has therapeutic potency, we first treated CT26 tumor cells with different concentrations of free PTC209, NPs-PTC209, or HA-NPs-PTC209, followed by the CCK-8 cell viability assay and IC50 calculation. As shown in Figure $\mathbf{S 2}$ and Table S3, compared with the free PTC209 and NPs-PTC209 treated groups, HA-NPs-PTC209 exhibits a significantly lower IC50 at both $24 \mathrm{~h}$ (approx. $759 \mathrm{nM}$ ) and $48 \mathrm{~h}$ (approx. $588 \mathrm{nM}$ ). These results indicate that HA-NPsPTC209 can potently inhibit the proliferation of CT26 tumor cells. We chose $250 \mathrm{nM}$ PCT209, which is lower than the IC50 for each formulation, to carry out subsequent in vitro experiments. Cancer stem cells tend to form multicellular spheres, whereas non-stem tumor cells grown in monolayers under conventional cell culture conditions. We then evaluated multicellular sphere formation after treatment with the different PTC209 formulations. Significant phenotypic changes were observed in CT26-derived multicellular spheres in all the PTC209-containing groups. Compared with the free PTC209 and NPs-PTC209 treatment groups, the HA-NPs-PTC209 group had spheres of the smallest diameters (Figure 4A\&C). We also examined the effects of different PTC209 formulations on the migration ability of CT26 colon cancer cells by a wound healing test. As shown in Figure 4B\&D, both NPs-PTC209 and HA-NP-PTC209 displayed a potent migration inhibiting efficiency. It should be noted that even the free PTC209 effectively reduced the migration of CT26 tumor cells. These data suggest that HA-NP-PTC209 can significantly suppress the stemness of CT26 colon cancer cells.

To further explore the mechanism of stemness inhibition, we examined the expression of two nuclear markers of stemness (Oct-4 and Sox-2) $)^{34,35}$ in CT26 colon cancer cells after incubation with the different PTC209 formulations. We found that the expression of Oct-4 and Sox-2 were down-regulated after treatment with the equivalent of $250 \mathrm{nM}$ PTC209 of any of the formulations for $48 \mathrm{~h}$; Sox-2 expression was strongly decreased in the HA-NPs-PTC209 treated group (Figure 4E\&F). CD44 and CD133 are associated with clone formation efficiency and proliferative ability, respectively ${ }^{36}$. The levels of these two proteins were strongly reduced after PTC209 treatment, as determined by flow cytometry (Figure S3).

\section{In vivo distribution of HA-NPs-PTC209}

We first established a stable luciferase-expressing CT26 cell line by virus transduction. Next, we used this cell line to develop an orthotopic colon tumor model. Briefly, tumor cells suspended in PBS with 
matrigel and India ink were inoculated surgically into the cecum wall (Figure S4). Monitoring of the luciferase activity over the course of 20 days verified that we had successfully established an orthotopic colon cancer model (Figure S5). H\&E staining of tumor tissue revealed the primary tumor invades into lumen of cecum tissue and metastatic tumor invades to the circular muscle in gastrointestinal system (Figure S6). The success rate of this method was about $80 \%$. In order to investigate the in vivo gut distribution of HA-NPs-PTC209, we treated fasting BALB/c mice, orally, with Cy5.5-labled NPsPTC209 (NPs-PTC209-Cy5.5) or Cy5.5-labeled HA-NPs-PTC209 (HA-NPs-PTC209-Cy5.5). The fluorescence signal in the gut was examined ex vivo at 1, 4, 8, and $24 \mathrm{~h}$ post-administration using a fluorescence imaging system. As shown in Figure S7, the fluorescence was mainly present in the upper gastrointestinal tract within the first $4 \mathrm{~h}$. Over time, the Cy5.5 signal moved down to the cecum and colon after $8 \mathrm{~h}$. Based on these data, we chose $8 \mathrm{~h}$ and $24 \mathrm{~h}$ post administration to conduct the following experiments. To assess the tumor targeting ability of HA-NPs-PTC209, mice with orthotopic colon tumors were treated orally with NPs-PTC209-Cy5.5 or HA-NPs-PTC209-Cy5.5. We excised the tumor-bearing colons $8 \mathrm{~h}$ and $24 \mathrm{~h}$ later and examined them by fluorescence imaging. In contrast to the non-targeted group, the HA-NPs-PTC209-Cy5.5 colons exhibited a stronger fluorescence signal at the tumor site. Importantly, we observed the highest signal intensity in the tumor after treatment for $24 \mathrm{~h}$ (Figure 5A\&B). The tumor tissues were also sectioned and observed by confocal laser scanning microscopy to further verify the targeting capacity of HA-NPs-PTC209-Cy5.5. The HA-NPs-PTC209-Cy5.5 treated group exhibited the strongest signal intensity not only at $8 \mathrm{~h}$ post oral administration but also at the $24 \mathrm{~h}$ timepoint (Figure 5C). These results suggest that HA-modified NPs improve the penetrance and accumulation of the nanoparticles in tumor tissues. Consistent with the results of the fluorescence experiments, the content of PTC209 in HA-NPs-PTC209-treated tumor tissue was also the highest (Figure 5D). Intriguingly, the amount of PTC209 in the small intestine and colon tissues was also higher in the NPs-PTC209 and HANPs-PTC209 groups compared with the free drug treatment groups, indicating that nanoparticle formulations themselves also improve the PTC209 pharmacokinetic properties.

\section{In vivo antitumor effects of HA-NPs-PTC209}

So far, we have verified that HA-NPs-PTC209 can efficiently enhance the penetrance and accumulation of drug in tumor tissue. We next evaluated the in vivo antitumor efficacy of HA-NPs-PTC209 in the CT26 orthotopic tumor-bearing mouse model. Five days after inoculation, the mice were randomized 
into 5 groups and treated with saline, empty NPs, free PTC209, NPs-PTC209 or HA-NPs-PTC209 (equivalent to $30 \mathrm{mg} / \mathrm{kg}$ bodyweight of PTC209) every two days for 7 treatments (Figure 6A). The growth of the tumors was monitored by bioluminescence detection. Compared with the saline and NPs groups, the PTC209, NPs-PTC209 and HA-NPs-PTC209 treatment groups remarkably restrained the rapid tumor growth (Figure 6B\&D). Notably, the HA-NPs-PTC209 group showed the best therapeutic effects than the other formulations. Additionally, the average number of metastases per gastrointestinal tract was significantly decreased in all PTC209-containing groups. Consistent with the growth rate curve, the HA-NPs-PTC209 treated group exhibited the fewest gastrointestinal metastases (Figure 6C\&E). Taken together these results indicate HA-NPs-PTC209 exerts a highly efficient anti-tumor effect. To explore the in vivo anti-tumor mechanisms of HA-NPs-PTC209, we analyzed the expression levels of Oct-4 and Sox-2 in the different treatment groups by immunohistochemistry and western blot analysis. The results in Figure 7A-C demonstrate that HA-NPs-PTC209 indeed significantly down-regulates the expression of both proteins, suggesting that HA-NPs-PTC209 prevents the progression of colon cancer in the orthotopic tumor model by reversing the stemness of CSCs.

\section{Tolerability of HA-NPs-PTC209}

To investigate the potential side effects of HA-NPs-PTC209, we first evaluated the in vitro cytotoxicity of empty HA-NPs at various concentrations. No apparent toxic effects were observed after incubation for $24 \mathrm{~h}$ and $48 \mathrm{~h}$ in CT26 tumor cells (Figure S8). We next examined the safety of HA-NPs-PTC209 in normal BALB/c mice. The animals were randomly divided into five groups and administered different drug formulations every 2 days for 5 treatments. Over the course of the treatment process, the body weights did not significantly change in any treatment group (Figure S9). In addition, we did not observe any histological changes in the kidney, liver, spleen, stomach or intestine, suggesting that HA-NPsPTC209 is well-tolerated by the digestive organs (Figure S10).

\section{Conclusion}

In the present study, we have developed an orally administered, colon cancer-targeting nanoparticle for the delivery of the BMI-1 inhibitor, PTC209. Functionalized with the CD44 / CD168-targeting ligand, 
HA, the nanoparticles accumulate in orthotopic colon tumors to reverse CSC stemness. Our data demonstrate that the nanoparticle exhibits considerable in vitro stability, while the HA decoration outside the nanoparticle endows it with an excellent tumor-targeting capacity. This simple strategy also significantly inhibited clone formation and migration of colon cancer cells in vitro, and suppressed in vivo progression of primary tumors and metastasis by reversing stemness. We believe our approach to be a promising and universally applicable strategy for colon cancer treatment or for combination with other therapeutic regimens (such as radiotherapy, chemotherapeutics and immunotherapy).

Although our formulation is effective for colon cancer therapy, there are still several obstacles to overcome before translation to the clinic. Our results indicate that the small intestine is the main tissue for absorption of the nanoparticles. To enhance colon accumulation, different forms of enteric-protective technologies such as microencapsulation, hydrogel or nanofibers, could be used to help NPs escape from the harsh small intestinal environment ${ }^{23,37-39}$. The suitable design for NPs could not only enhance the retention of the payload in orthotopic colon tissues but also be free of systemic side effects. Additionally, these NPs should be investigated in additional colon cancer models, such as chemically-induced or genetically modified CRC models. Finally, future studies should examine whether combination therapy can potentiate the efficacy of this nanoparticle formulation.

\section{Acknowledgements}

We thank Dr. Jian You from Zhejiang University for help with the CT26-luc establishment. This work was supported by grants from the National Basic Research Plan of China (2018YFA0208900), the National Natural Science Foundation of China (31661130152), the Academy of Medical Sciences-Newton Advanced Fellowship (NAF003\1002), the National Postdoctoral Program for Innovative Talents (BX20180083) and the China Postdoctoral Science Foundation (2018M640100).

\section{Appendix A. Supplementary data}

Supplementary data related to this article can be found at: 


\section{References}

1. M. Arnold, M. S. Sierra, M. Laversanne, I. Soerjomataram, A. Jemal, F. Bray, Global patterns and trends in colorectal cancer incidence and mortality, Gut 66 (4) (2017) 683-691.

2. P. Li, H. Wu, H. Zhang, Y. Shi, J. Xu, Y. Ye, D. Xia, J. Yang, J. Cai, Y. Wu, Aspirin use after diagnosis but not prediagnosis improves established colorectal cancer survival: a meta-analysis, Gut 64 (9) (2015) 1-7.

3. A. S. A. Lila, N. Moriyoshi, M. Fukushima, C. Huang, H. Wada, T. Ishida, Metronomic S-1 dosing and thymidylate synthase silencing have synergistic antitumor efficacy in a colorectal cancer xenograft model, Cancer Lett. 400 (2017) 223-231.

4. M. S. Wicha, S. Liu, G. Dontu, Cancer Stem Cells: An Old Idea-A Paradigm Shift, Cancer Res. 66 (4) (2006) 1883-1890

5. H. Clevers, The cancer stem cell: premises, promises and challenges, Nat. Med. 17 (3) (2011) 313 319.

6. E. Batlle, H. Clevers, Cancer stem cells revisited, Nat. Med. 23 (10) (2017) 1124-1134.

7. H. Korkaya, M. S. Wicha, HER2 and Breast Cancer Stem Cells: More than Meets the Eye, Cancer Res. 73 (12) (2013) 3489-3493.

8. M. Todaro, M. G. Francipane, J. P. Medema, G. Stassi, Colon Cancer Stem Cells: Promise of Targeted Therapy, Gastroenterology 138 (6) (2011) 2151-2162.

9. M. Ahmed, K. Chaudhari, R. Babaei - Jadidi, L. V. Dekker, A. Shams Nateri, Emerging Drugs Targeting Epithelial Cancer Stem-Like Cells, Stem Cells 35 (4) (2017) 839-850.

10. A. Kreso, P. van Galen, N. M. Pedley, E. Lima-Fernandes, C. Frelin, T. Davis, L. Cao, R. Baiazitov, W. Du, N. Sydorenko, Y. C. Moon, L. Gibson, Y. Wang, C. Leung, N. N. Iscove, C. H. Arrowsmith, E. Szentgyorgyi, S. Gallinger, J. E. Dick, C. A. O’Brien, Self-renewal as a therapeutic target in human colorectal cancer, Nat. Med. 20 (1) (2014) 29-36.

11. A. Bolomsky, K. Schlangen, W. Schreiner, N. Zojer, H. Ludwig, Targeting of BMI-1 with PTC-209 shows potent anti-myeloma activity and impairs the tumour microenvironment, J. Hematol. Oncol. 9 (17) (2016) $1-13$

12. L. Mourgues, V. Imbert, M. Nebout, P. Colosetti, Z. Neffati, P. Lagadec, E. Verhoeyen, C. Peng, E. Duprez, L. Legros, N. Rochet, V. Maguer-Satta, F. E. Nicolini, D. Mary, J. F. Peyron, The BMI1 
polycomb protein represses cyclin G2-induced autophagy to support proliferation in chronic myeloid leukemia cells, Leukemia 29 (10) (2015) 1993-2002.

13. H. R. Siddique, M. Saleem, Role of BMI1, a Stem Cell Factor, in Cancer Recurrence and Chemoresistance: Preclinical and Clinical Evidences, Stem Cells 30 (3) (2012) 372-378.

14. J. Du, Y. Li, J. Li, J. Zheng, Polycomb group protein Bmil expression in colon cancers predicts the survival, Med. Oncol. 27 (4) (2010) 1273-1276.

15. J. E. Visvader, G. J. Lindeman, Cancer stem cells in solid tumours: accumulating evidence and unresolved questions, Nat. Rev. Cancer 8 (10) (2008) 755-68.

16. S. Mitragotri, P. A. Burke, R. Langer, Overcoming the challenges in administering biopharmaceuticals: formulation and delivery strategies, Nat. Rev. Drug Discov. 13 (9) (2014) 655-672.

17. S. Mazzaferro, K. Bouchemal, G. Ponchel, Oral delivery of anticancer drugs III: formulation using drug delivery systems, Drug Discov. Today 18 (1-2) (2013) 99-104.

18. P. Lin, Y. Chiu, J. Huang, E. Chuang, F. Mi, K. Lin, J. Juang, H. Sung, K. W. Leong, Oral Nonviral Gene Delivery for Chronic Protein Replacement Therapy, Advanced Science 5 (8) (2017) 1701079

19. D. Shao, M. Li, Z. Wang, X. Zheng, Y. H. Lao, Z. Chang, F. Zhang, M. Lu, J. Yue, H. Hu, H. Yan, L. Chen, W. F. Dong, K. W. Leong, Bioinspired Diselenide-Bridged Mesoporous Silica Nanoparticles for Dual-Responsive Protein Delivery, Adv. Mater. 30 (2018) 1801198

20. Y. Zhang, J. Wei, J. Xu, W. S. Leong, G. Liu, T. Ji, Z. Cheng, J. Wang, J. Lang, Y. Zhao, L. You, X. Zhao, T. Wei, G. J. Anderson, S. Qi, J. Kong, G. Nie, Suppression of Tumor Energy Supply by Liposomal Nanoparticle-Mediated Inhibition of Aerobic Glycolysis, ACS Appl. Mater. Interfaces 10 (3) (2018) 2347-2353

21. L. Kotelevets, E. Chastre, J. Caron, J. Mougin, G. Bastian, A. Pineau, F. Walker, T. Lehy, D. Desmaele, P. Couvreur, A squalene-based nanomedicine for oral treatment of colon cancer, Cancer Res. 77 (11) (2017) 2964-2975.

22. K. Roy, H. Q. Mao, S. K. Huang, K. W. Leong, Oral gene delivery with chitosan-DNA nanoparticles generates immunologic protection in a murine model of peanut allergy, Nat. Med. 5 (4) (1999) $387-391$

23. B. Xiao, E. Viennois, Q. Chen, L. Wang, M. K. Han, Y. Zhang, Y. Kang, Y. Wan, D. Merlin, Silencing of Intestinal Glycoprotein CD98 by Orally Targeted Nanoparticles Enhances Chemosensitization of Colon Cancer, ACS Nano 12 (6) (2018) 5253-5265. 
24. D. S. Wilson, G. Dalmasso, L. Wang, S. V. Sitaraman, D. Merlin, N. Murthy, Orally delivered thioketal nanoparticles loaded with TNF-siRNA target inflammation and inhibit gene expression in the intestines, Nat. Med. 9 (11) (2010) 923-928

25. J. Huang, H. Zhang, Y. Yu, Y. Chen, D. Wang, G. Zhang, G. Zhou, J. Liu, Z. Sun, D. Sun, Y. Lu, Y. Zhong, Biodegradable self-assembled nanoparticles of poly (D, L -lactide-coglycolide) / hyaluronic acid block copolymers for target delivery of docetaxel to breast cancer, Biomaterials 35 (1) (2014) 550-566.

26. Y. Leng, A. Abdulah, M. K. Wendt, S. Calve, Hyaluronic acid, CD44 and RHAMM regulate myoblast behavior during embryogenesis, Matrix Biol. 78-79 (2018) 236-254

27. K. Y. Choi, H. S. Han, E. S. Lee, J. M. Shin, B. D. Almquist, D. S. Lee, J. H. Park. Hyaluronic Acid-Based activatable Nanomaterials for stimuli-responsive imaging and Therapeutics: Beyond CD44-Mediated drug delivery, Adv. Mater. (2019) 1803549

28. M. Zhang, C. Xu, L. Wen, M. K. Han, B. Xiao, J. Zhou, Y. Zhang, Z. Zhang, E. Viennois, D. Merlin, A hyaluronidase responsive nanoparticle-based drug delivery system for targeting colon cancer cells, Cancer Res. 76 (24) (2016) 7208-7218.

29. H. Wang, Y. Zhao, Y. Wu, Y. L. Hu, K. Nan, G. Nie, H. Chen, Enhanced anti-tumor efficacy by co-delivery of doxorubicin and paclitaxel with amphiphilic methoxy PEG-PLGA copolymer nanoparticles, Biomaterials 32 (32) (2011) 8281-8290.

30. J. Lang, X. Zhao, X. Wang, Y. Zhao, Y. Li, R. Zhao, K. Cheng, Y. Li, X. Han, X. Zheng, H. Qin, M. Geranpayehvaghei, J. Shi, G. J. Anderson, J. Hao, H. Ren, G. Nie, Targeted Co-delivery of the Iron Chelator Deferoxamine and a HIF1 $\alpha$ Inhibitor Impairs Pancreatic Tumor Growth, ACS Nano 13 (2) (2019) 2176-2189

31. K. Narumi, T. Udagawa, A. Kondoh, A. Kobayashi, H. Hara, Y. Ikarashi, S. Ohnami, F. Takeshita, T. Ochiya, T. Okada, M. Yamagishi, T. Yoshida, K. Aoki, In vivo delivery of interferon-gene enhances tumor immunity and suppresses immunotolerance in reconstituted lymphopenic hosts, Gene ther. 19 (1) (2011) 34-48

32. K. P. Terracina, T. Aoyagi, W. C. Huang, M. Nagahashi, A. Yamada, K. Aoki, K. Takabe, Development of a metastatic murine colon cancer model, J. Surg. Res. 199 (1) (2015) 106-114.

33. E. L. Mcconnell, H. M. Fadda, A. W. Basit, Gut instincts: Explorations in intestinal physiology and drug delivery, Int. J. Pharm. 364 (2) (2008) 213-226. 
34. I. H. Park, R. Zhao, J. A. West, A. Yabuuchi, H. Huo, T. A. Ince, P. H. Lerou, M. W. Lensch, G. Q. Daley, Reprogramming of human somatic cells to pluripotency with defined factors, Nature 451 (7175) (2008) 141-146.

35. S. Saigusa, K. Tanaka, Y. Toiyama, T. Yokoe, Y. Okugawa, Y. Loue, C. Miki, M. Kusunoki, Correlation of CD133, OCT4, and SOX2 in Rectal Cancer and Their Association with Distant Recurrence After Chemoradiotherapy, Ann. Surg. Oncol. 16 (12) (2009) 3488-3498.

36. Z. Zhang, X. Bu, H. Chen, Q. Wang, W. Sha, Bmi-1 promotes the invasion and migration of colon cancer stem cells through the downregulation of E-cadherin, Int. J. Mol. Med. 38 (4) (2016) 11991207.

37. P. Tipduangta, P. Belton, L Fabian, L. Y. Wang, H. Tang, M. Eddleston, S. Qi, Electrospun Polymer Blend Nanofibers for Tunable Drug Delivery: The Role of Transformative Phase Separation on Controlling the Release Rate, Mol. Pharm. 13 (1) (2016) 25-39.

38. L. A. Sharpe, J. E. Vela Ramirez, O. M. Haddadin, K. A. Ross, B. Narasimhan, N. A. Peppas, pHResponsive Microencapsulation Systems for the Oral Delivery of Polyanhydride Nanoparticles, Biomacromolecules 19 (3) (2018) 3904-3904.

39. D. Han, J. S. Andrew, Selective pH-Responsive Core-Sheath Nanofiber Membranes for Chem/Bio/Med Applications: Targeted Delivery of Functional Molecules, ACS Appl. Mater. Interfaces, 9 (49) (2017) 42653-42660 
A
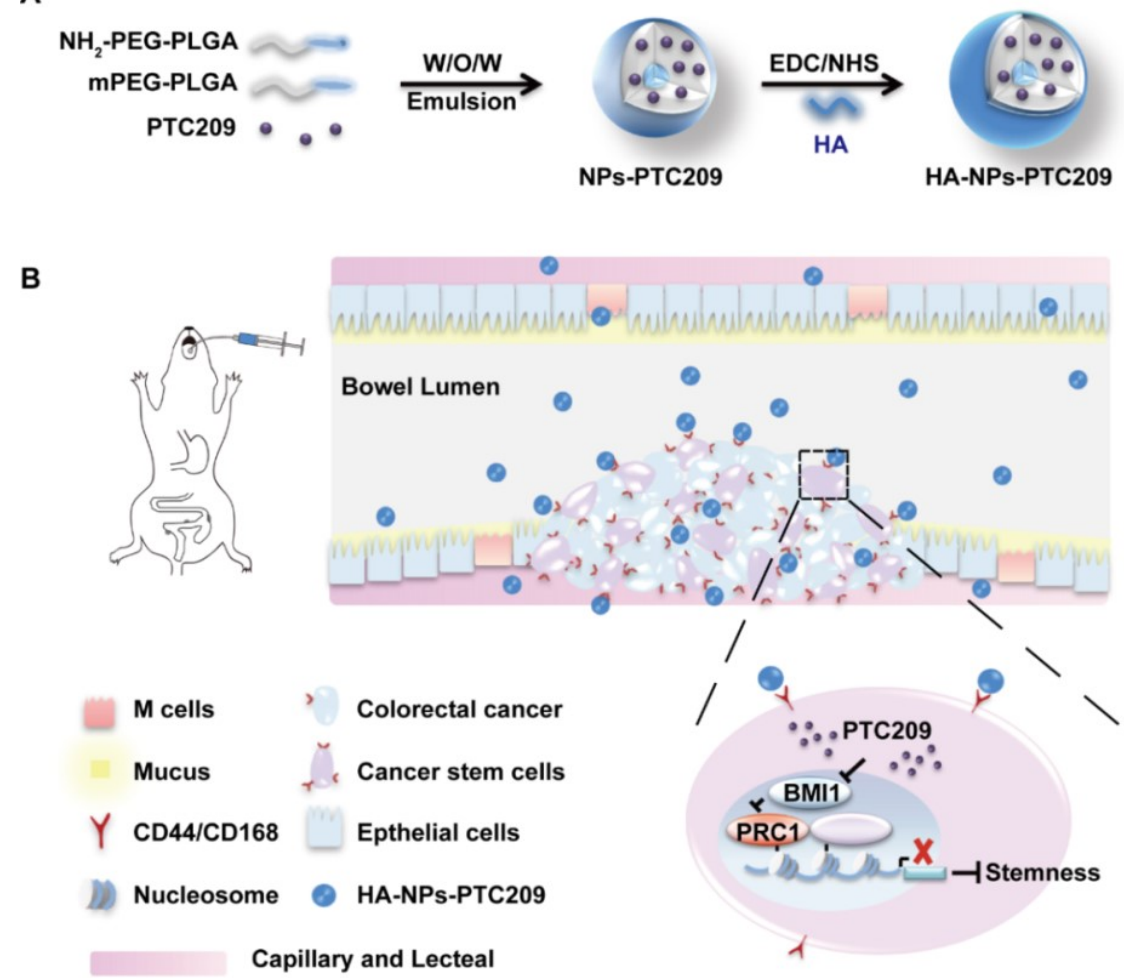

Figure 1. Schematic diagram of preparation and proposed mechanism of action of HA-NPs-

PTC209 in orthotopic colon tumor model. (A) PTC209-loaded NPs were assembled from $\mathrm{NH}_{2}$-PEGPLGA and mPEG-PLGA copolymer by a double emulsion method, after which hyaluronic acid (HA) modified the surface of the NPs by an EDC-NHS reaction. (B) Proposed mechanism of HA-NPs-PTC209 action. After oral treatment, HA-NPs-PTC209 actively target CSCs in colorectal cancer (CRC) and are internalized. Consequently, the released PTC209 inhibits BMI-1 and down-regulates the expression of stemness-related proteins to lower tumor stemness and recurrence. 

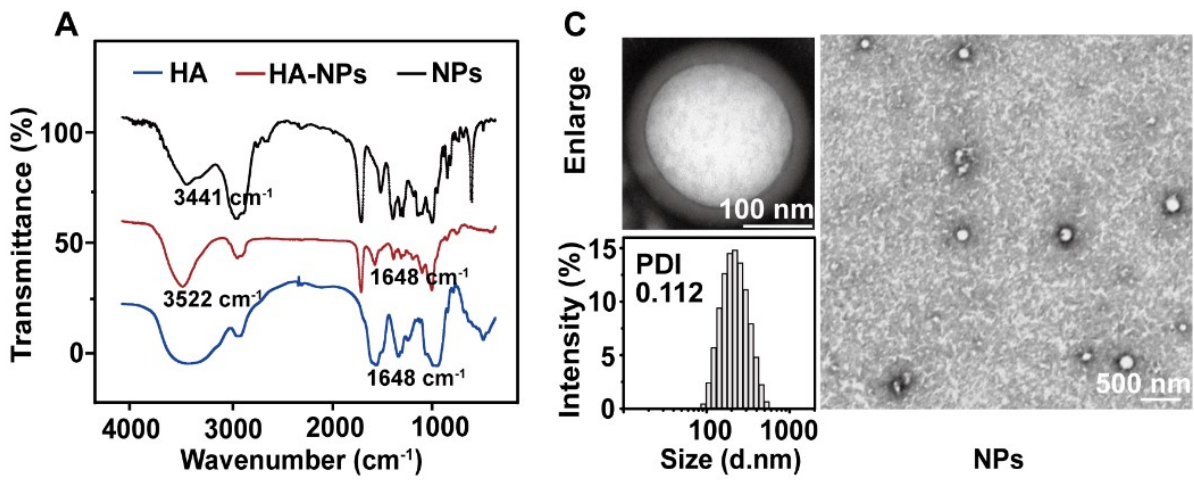

B

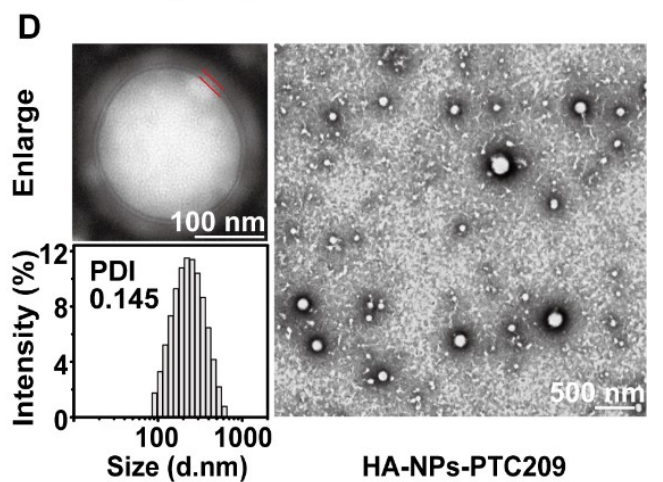

Figure 2. Physicochemical characterization of the NPs and HA-NPs-PTC209. (A) FT-IR spectra of HA, NPs and HA-NPs. (B) Average hydrodynamic diameter and surface zeta potential of bare NPs, HANPs and HA-NPs-PTC209. The data are presented as the mean \pm SD, $n=3$. (C-D) Transmission electron microscopy and dynamic light scattering characterization of NPs (C) and HA-NPs-PTC209 (D). Representative TEM image of HA-NPs-PTC209, a $5 \mathrm{~nm}$ layer of HA on the surface was observed. 
A
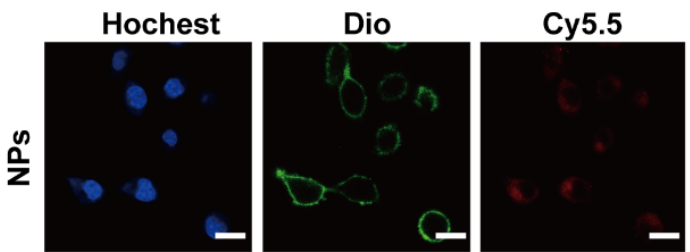

Merge
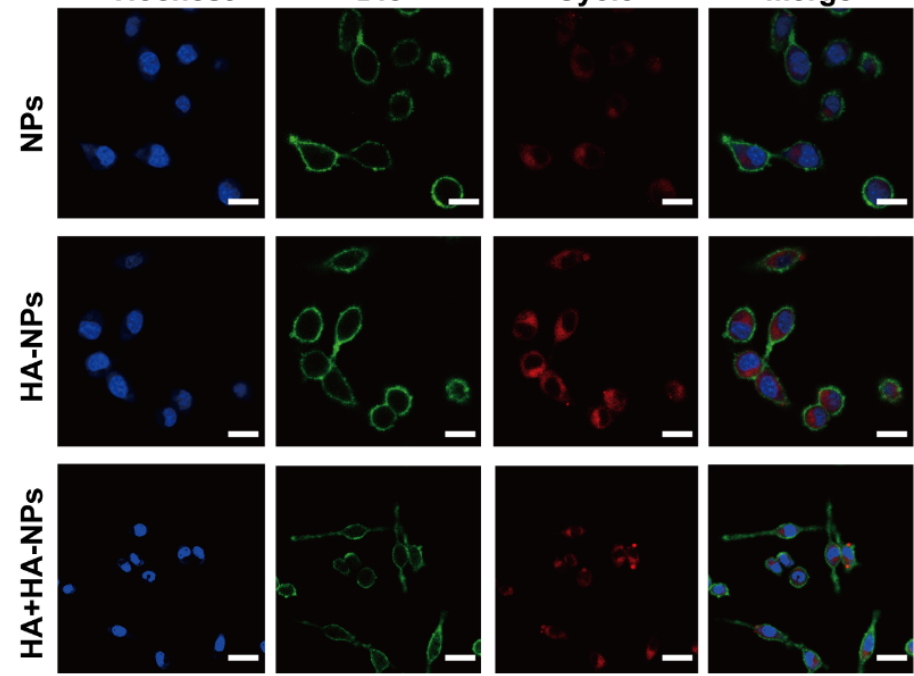

B
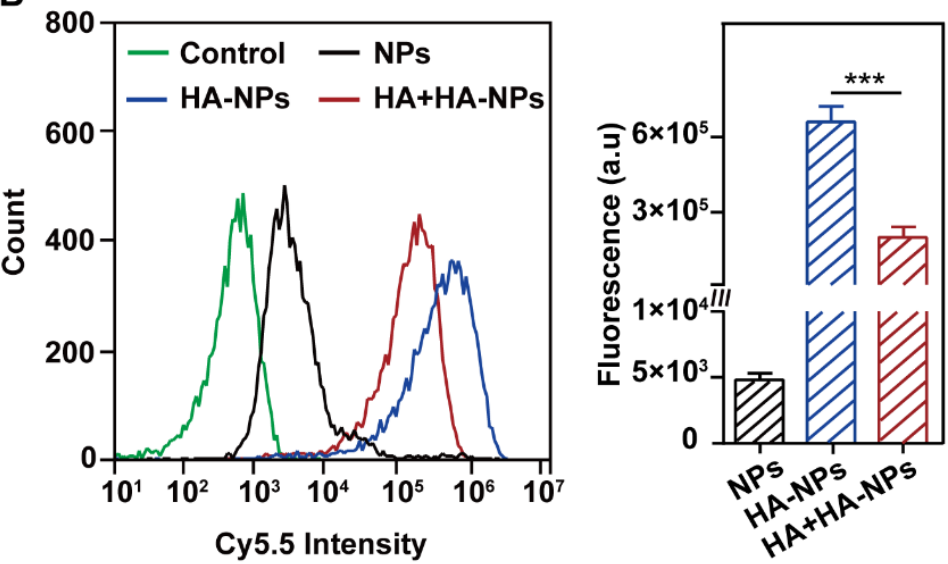

Figure 3. Evaluation of HA-NPs in vitro targeting capacity. (A) Fluorescence microscopy images of cellular targeting profiles of Cy5.5-labled HA-NPs in CT26 cells after treatment for $4 \mathrm{~h}$. The nucleus was stained with Hoechst (blue), the membrane was stained with Dio (green). Scale bars are $20 \mu \mathrm{m}$. (B) Flow cytometry of internalized or targeted Cy5.5-labled HA-NPs (left) and quantification (right) after $2 \mathrm{~h}$ incubation. The data are presented as the mean $\pm \mathrm{SD}, \mathrm{n}=3(* * * p<0.001)$. 
A

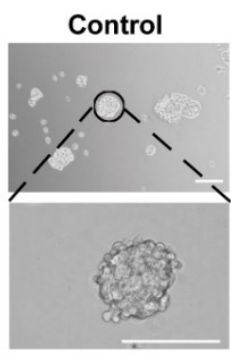

NPs

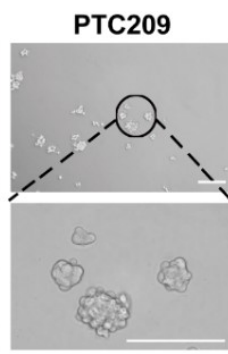

NPs-PTC209

HA-NPs-PTC209

B
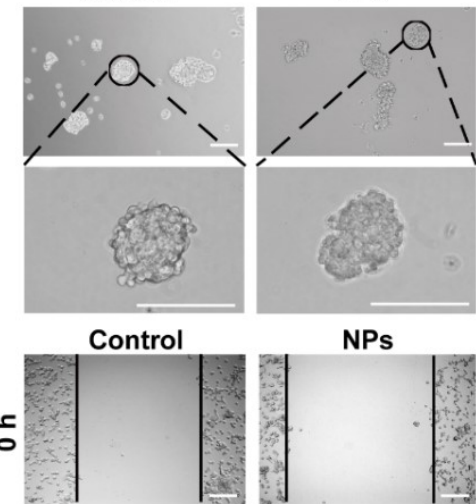

NPs

PTC209
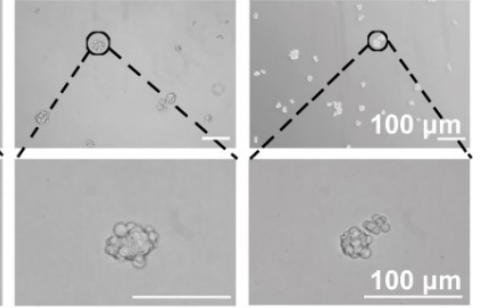

NPs-PTC209

HA-NPs-PTC209
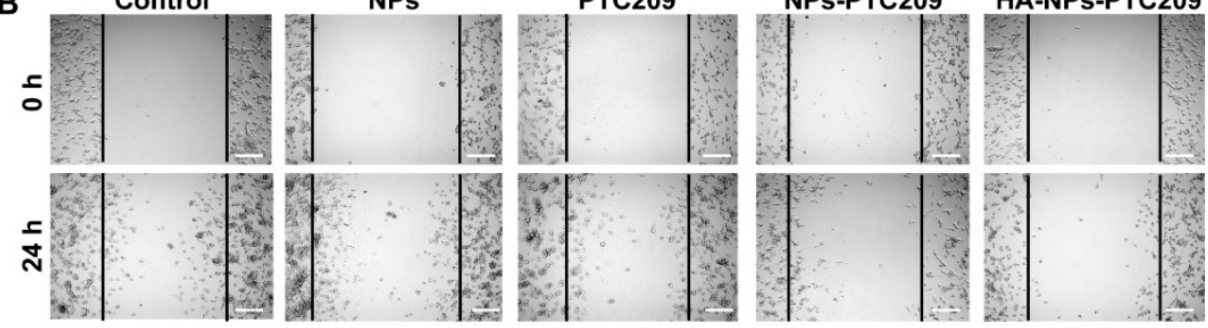

C

D
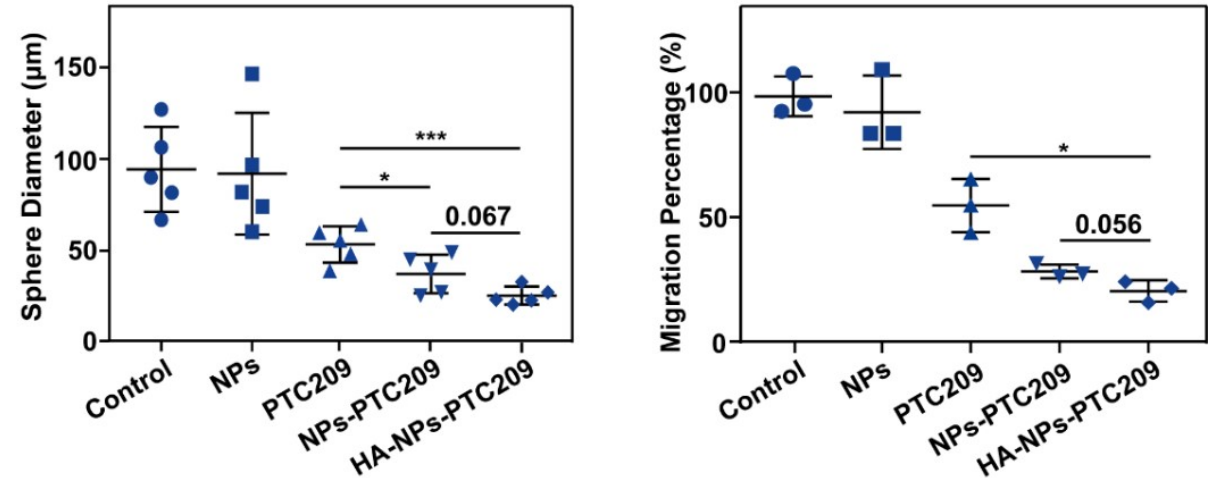

E
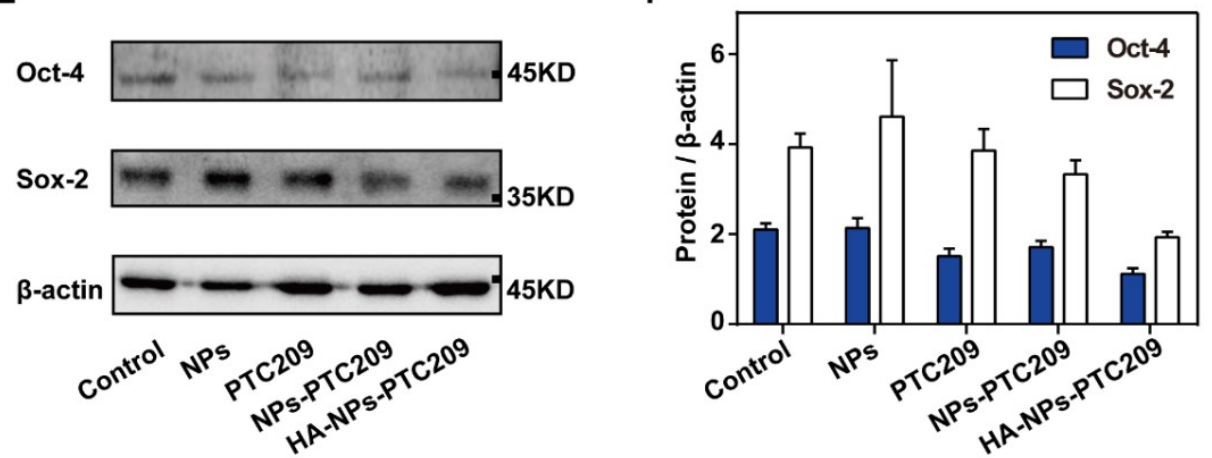

Figure 4. In vitro stemness reversion of colon cancer cells. (A) Clone formation assay of CT26 cancer cells after exposure to the indicated formulations of PTC209 for 7 days. (B) A would heal scratch assay was carried out to evaluate the migration capability of CT26 cancer cells after a $24 \mathrm{~h}$ incubation. Scale bars are $100 \mu \mathrm{m}$. (C) Quantification of CT26 sphere diameters in the experiment shown in panel A. (D) The percentage of migration in the experiment presented in panel B. (E) Western blot analysis of Oct-4 and Sox-2 in CT26 cells exposed to the indicated formulations of PTC209 for $48 \mathrm{~h}$. The concentration of PTC209 was $250 \mathrm{nM}$ in these experiments. (F) Quantification of the results of the experiment presented in panel E. The data are represented as the mean $\pm \mathrm{SD}(* p<0.05, * * * p<0.001)$. 
A

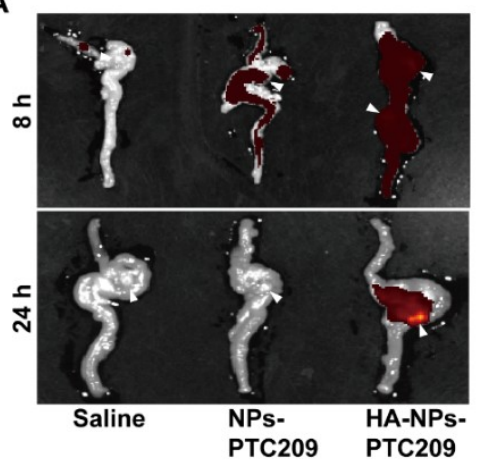

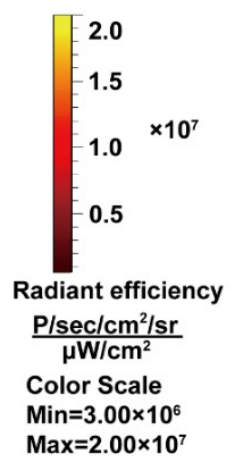

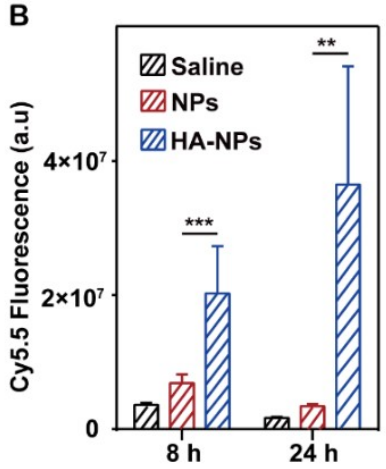

C
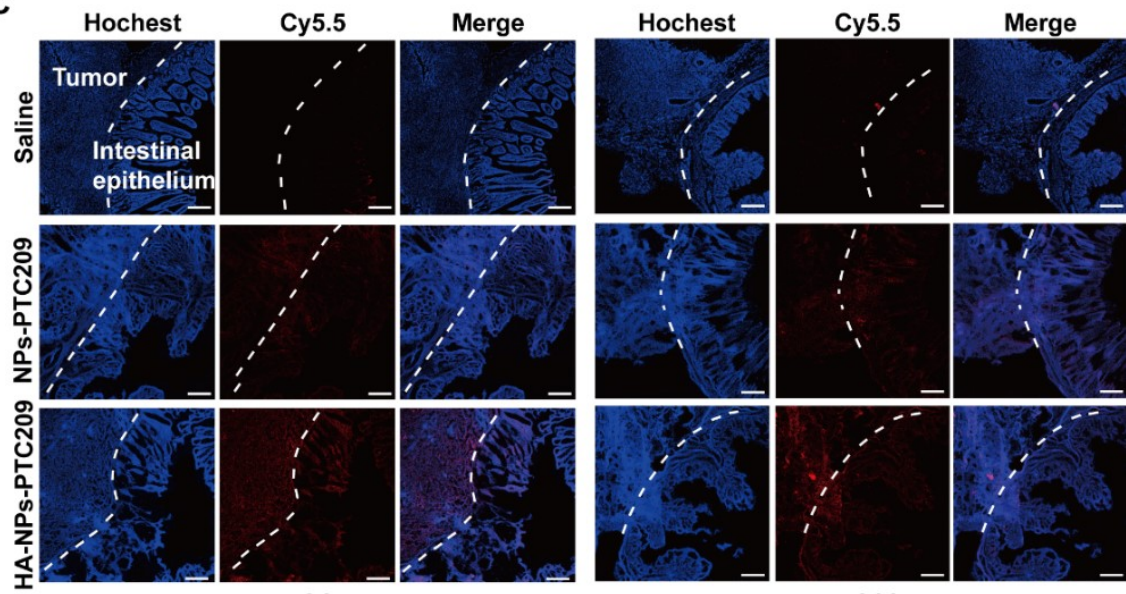

$24 \mathrm{~h}$
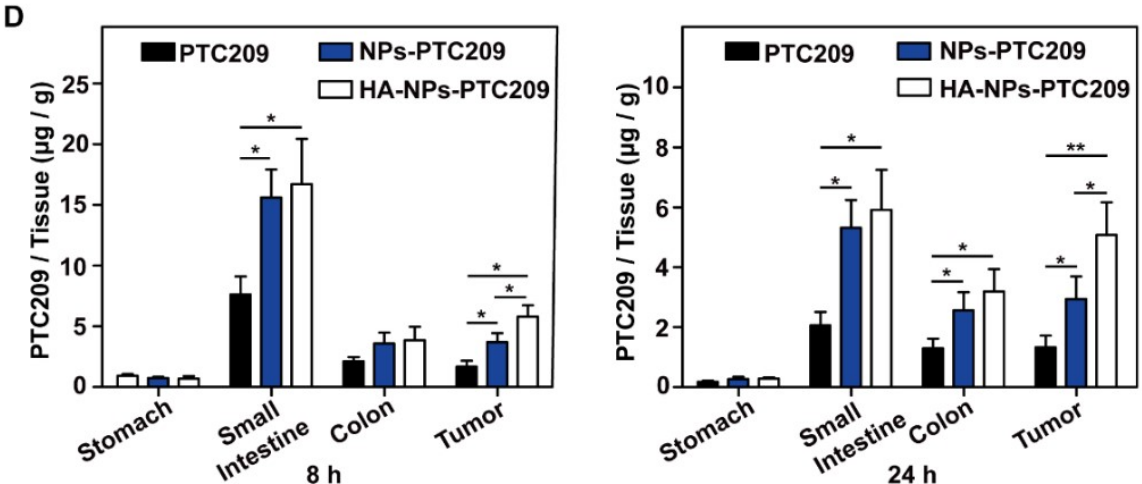

Figure 5. Ex vivo tumor targeting activity of HA-NPs-PTC-209. (A) Ex vivo fluorescence images of Cy5.5-labled NPs-PTC-209 and HA-NPs-PTC-209 in tumor-bearing cecum-colon organs after oral administration for $8 \mathrm{~h}$ and $24 \mathrm{~h}$. The white arrows point to tumors in the cecum-colon site. (B) Quantification of the fluorescence signals in the experiment presented in panel A. (C) Representative confocal images of frozen sections from the excised cecum-colon organs. The nuclei are stained with Hoechst (blue). Scale bars are $200 \mu \mathrm{m}$. (D) The amounts of PTC-209 in the tumor and digestive organs were quantified in the indicated treatment groups $8 \mathrm{~h}$ and $24 \mathrm{~h}$ post administration, as determined by HPLC. The dose of PTC209 was equivalent to $30 \mathrm{mg} / \mathrm{kg}$. The data are represented as the mean $\pm \mathrm{SD}, \mathrm{n}=3\left({ }^{*} p<0.05,{ }^{* *} p\right.$ $<0.01, * * * p<0.001)$. 
A

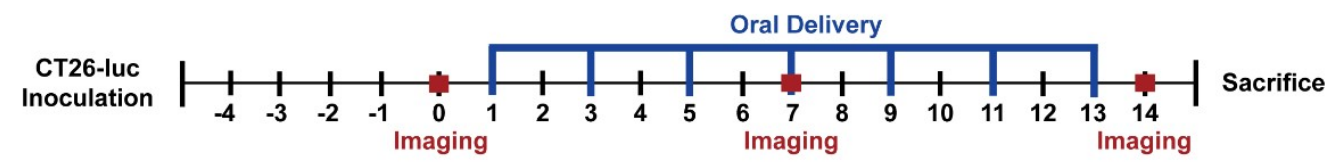

B

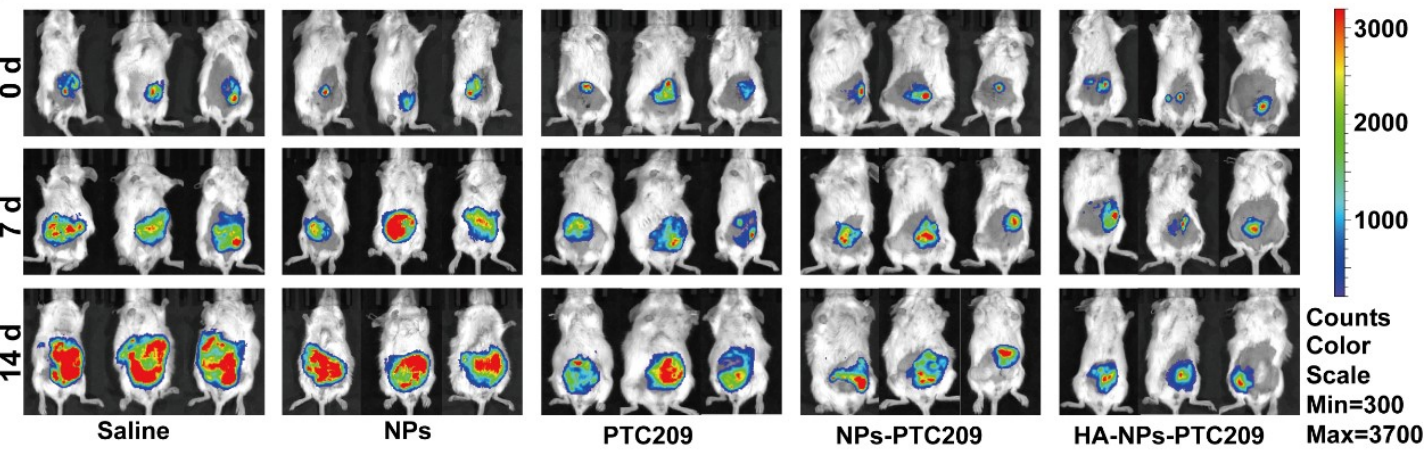

C

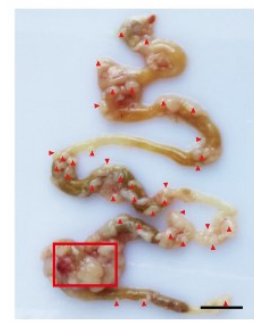

Saline

D

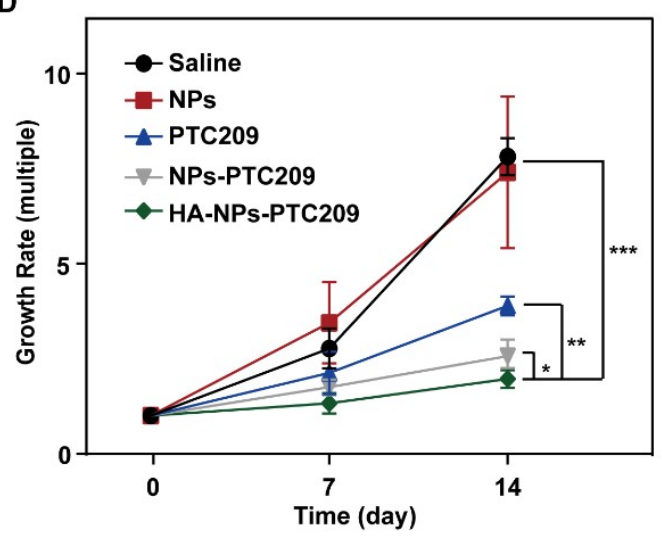

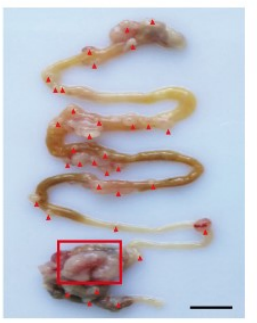

NPs

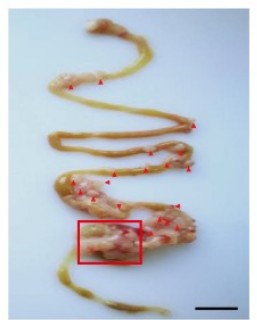

PTC209

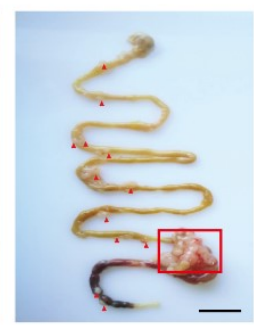

NPs-PTC209

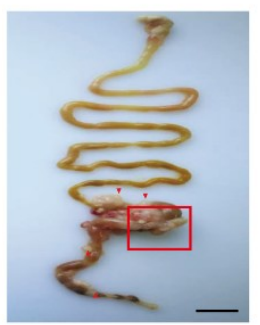

HA-NPs-PTC209

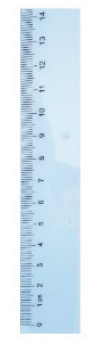

E

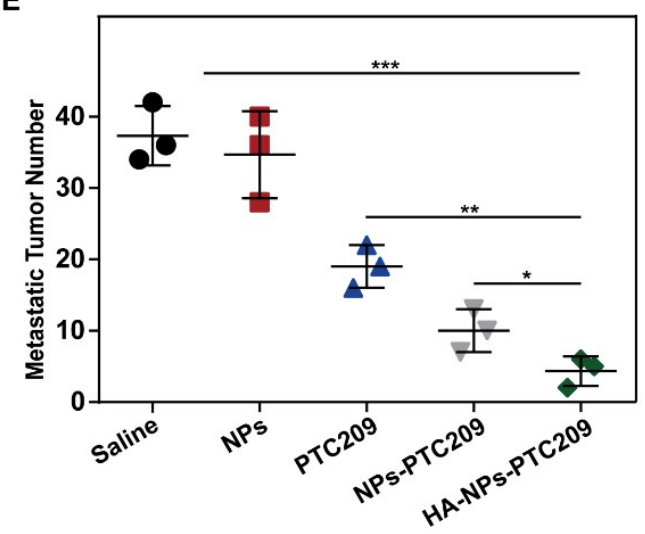

Figure 6. In vivo antitumor activity of HA-NPs-PTC-209. (A) Experimental timeline for the drug treatment groups. (B) In vivo bioluminescence imaging of CT26 tumor-bearing mice. Three representative mice per group are presented. (C) Representative gastrointestinal samples from the indicated treatment groups. Red arrows point to metastatic tumors in the gastrointestinal system and primary tumors were marked in red rectangular box. Scale bars are $2 \mathrm{~cm}$. (D) The relative quantification of the bioluminescence in the tumors from the indicated treatment groups on the $0^{\text {th }}, 7^{\text {th }}$ and $14^{\text {th }}$ days. (E) Quantitative analysis of the metastatic tumors in the gastrointestinal tissue results presented panel C. The data are presented as the mean $\pm \mathrm{SD}, \mathrm{n}=3\left({ }^{*} p<0.05,{ }^{* *} p<0.01\right.$ and $\left.{ }^{* * *} p<0.001\right)$. 

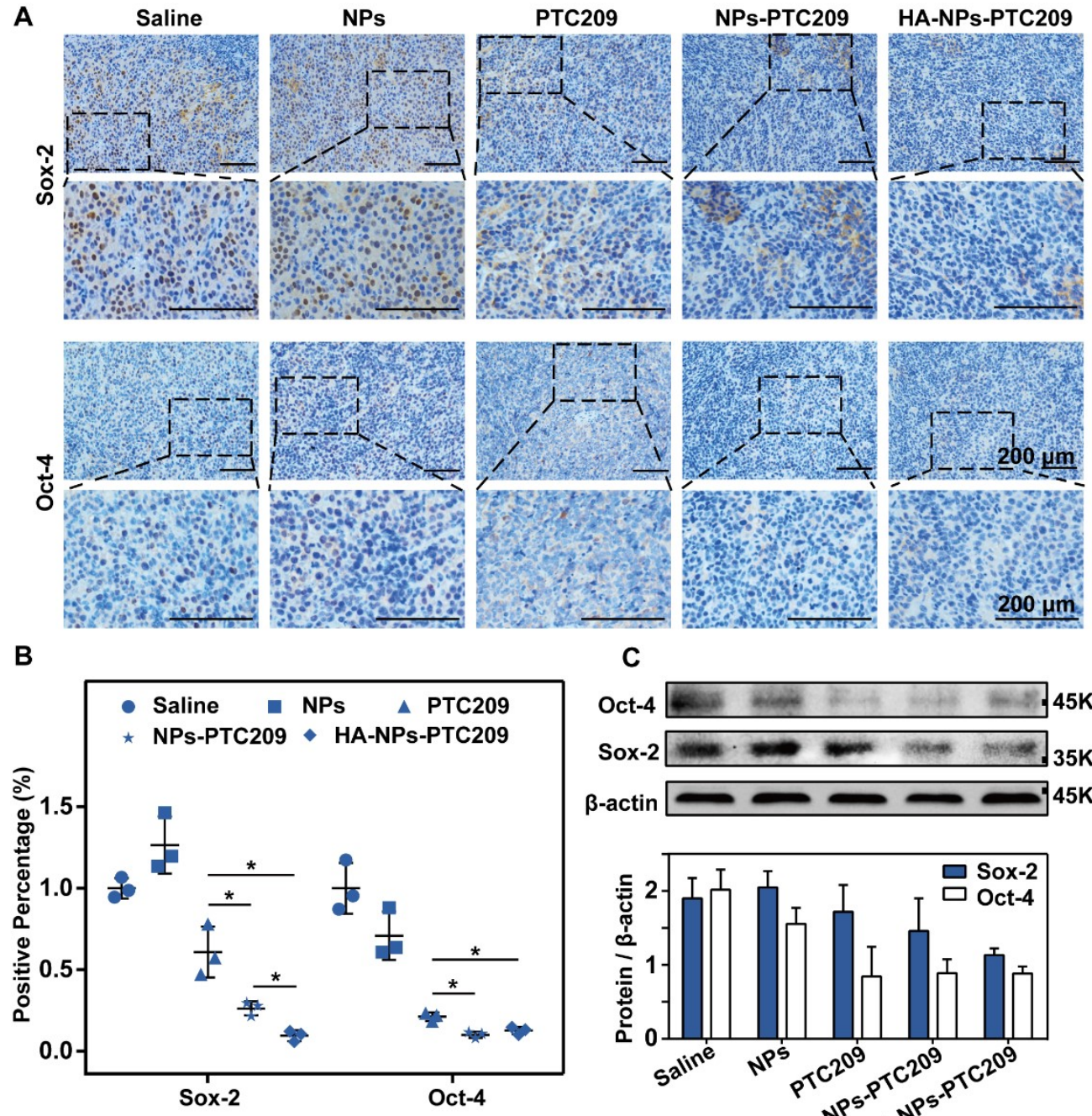

C
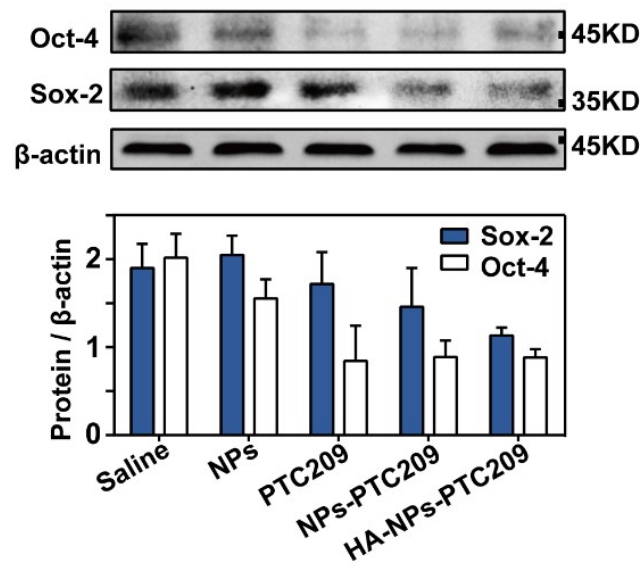

Figure 7. In vivo stemness reversion in the colon cancer tumor model. (A) Immunohistochemical analysis of Sox-2 and Oct-4 levels in the indicated PTC209 formulation treatment groups. (B) The percentage of Sox-2-and Oct-4 positive tumor cells from the experiment presented in panel A were quantified. Three fields were randomly selected for quantification, ${ }^{*} p<0.05,{ }^{* *} p<0.01$ and ${ }^{* * *} p<0.001$. (C) Western blot analysis of Sox-2 and Oct-4 levels in tumor tissues from mice treated with the indicated drug formulations. 